\title{
Special Nuclear Material Detection Studies With The SMANDRA Mobile System
}

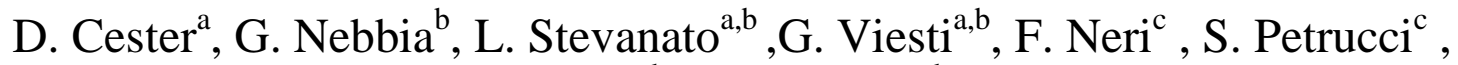 \\ S. Selmi ${ }^{\mathrm{c}}$,C. Tintori ${ }^{\mathrm{c}}$, P. Peerani ${ }^{\mathrm{d}}$, A. Tomanin ${ }^{\mathrm{d}}$ \\ ${ }^{a}$ Dipartimento di Fisica, Università di Padova, Via Marzolo 8, I-35131Padova, Italy \\ ${ }^{b}$ INFN Sezione di Padova, Via Marzolo 8, I-35131Padova, Italy \\ ${ }^{c}$ CAEN S.p.A., Via Vetraia 11, I-55049, Viareggio (LU), Italy \\ ${ }^{d}$ European Commission EC-JRC-ITU, I-21027 Ispra (VA), Italy
}

\begin{abstract}
The detection of special nuclear material has been studied with the SMANDRA mobile inspection system used both as a high sensitivity passive neutron/gamma spectroscopic tool and as an active inspection device using tagged neutrons. The detection of plutonium samples is possible with passive interrogation, the passive detection of uranium being much more difficult because of the low neutron yield and of the easiness of shielding the gamma rays. However, we show that active interrogation with tagged neutrons is able to provide signatures for the discrimination of uranium against other materials.
\end{abstract}

Keywords: Special Nuclear Material, mobile detection system, digital signal processing, neutron and gamma ray measurements

PACS: 29.40.-n, 27.90.+b, 28.20.-v , 89.20.Bb

\section{INTRODUCTION}

The SLIMPORT project is dedicated to the development of an integrated toolbox forming a complete security system for seaports. In this framework, a mobile inspection station (called SMANDRA, the Italian acronym stands for Sistema Mobile per Analisi Non Distruttive e RAdiometriche) has been developed. The aim of the SMANDRA system is to detect and identify sources of ionizing radiation or dangerous and/or illegal materials inside volumes tagged as "suspect" by conventional $\mathrm{X}$-ray scanners. The system is made of a passive and an active units. The passive unit includes gamma-ray and neutron detectors and hosts batteries, power supplies, frontend electronics and CPU. The active unit is equipped with a portable sealed neutron generator used to produce tagged neutron beams. The first unit can be used in standalone mode as a high efficiency spectroscopic radiometer. It can be used as well as detector package connected to the second unit for active interrogation of voxels inside a load using the Tagged Neutron Inspection System (TNIS) technique [1]. A complete description of the SMANDRA system is presented in [2]. Here we report on its application to the detection of special nuclear materials (SNM). 


\section{EXPERIMENTAL DETAILS}

In the SMANDRA system, photon spectroscopy is performed using a 2" $\mathrm{x} 2$ " BrilLanCe ${ }^{\mathrm{TM}} 380 \mathrm{LaBr}(\mathrm{Ce})$ detector and a $5 "$ x 5 " $\mathrm{NaI}(\mathrm{Tl})$ scintillator. The $\mathrm{LaBr}(\mathrm{Ce})$ detector offers the ultimate energy resolution but suffers from internal activity [3], thus showing some limitations in the detection and identification of weak ${ }^{40} \mathrm{~K}$ sources [4]. Moreover, these crystals are presently available only with modest volumes compared to other scintillators, therefore this entails limitations in the active mode operation when energetic gamma rays from inelastic excitation of oxygen and carbon nuclei need to be detected [5]. A large $\mathrm{NaI}(\mathrm{Tl})$ scintillator has been therefore selected to be used for energetic gamma rays in active investigations as well as high efficiency device in the detection and identification of weak gamma sources.

A ${ }^{3} \mathrm{He}$ proportional counter with a polyethylene moderator is a typical choice as neutron counter for systems operated in passive mode [6]. However, direct detection of fast neutrons both in passive and active mode is an important plus, as shown below, and imposes the use of a liquid organic scintillator. The interest in the detection of fast neutrons is motivated by the energy dependence of the neutron background [7]. In the SMANDRA system a 5" x 2" NE213 scintillator and an ASPECT SN-01 ${ }^{3} \mathrm{He}$ proportional counter were employed. The liquid scintillator was operated at very low threshold. However, the acceptance of a neutron event was obtained by energy windowing in order to avoid gamma-ray contamination and optimize the signal-tonoise ratio with respect to the neutron natural background. An important distinctive factor of the SMANDRA system is that both operating modes (passive and active) are managed by a CAEN VME electronic front end based on fast digitizers. The front end makes use of a prototype battery operated VME mini-crate with a Bridge USB V1718. The mini-crate hosts a HV system V6533 Programmable HV Power Supply (6 Ch., 4 kV, 3 mA, 9 W) and a V1720 8 Channel 12bit $250 \mathrm{MS} / \mathrm{s}$ Digitizer. Inside the V1720, Digital Pulse Processing (DPP) algorithms are implemented by using FPGA, providing on-line for each event a) a time stamp, b) a complete integration of the signal, c) a partial integration of the signal used for Pulse Shape Discrimination (PSD) in the liquid scintillator and d) the possibility of storing a selected part of the digitized signal.

Special Nuclear Material, Pu and U samples with different isotopic composition has been made available by the PERLA Laboratory at JRC Ispra where the tests were performed.

\section{DETECTION IN PASSIVE MODE}

As a first attempt, the detection of SNM samples was explored in passive mode. The sensitivity of the system in detecting weak gamma ray and neutron sources is reported in [2]. The detection protocol for the present SNM study started with the measurement of a background run in order to define the alarm thresholds for neutrons in the NE213 detector, as well as for gamma ray in the $\mathrm{NaI}(\mathrm{Tl})$ scintillator for 3 seconds measuring time. All SNM samples then were placed in front of the detector box, shielded by 6 $\mathrm{mm}$ of iron. The distances were adjusted so that the various sources would deliver a measured dose of $0.5 \mu \mathrm{Sv} / \mathrm{h}$ at the surface of the detector box. For all samples, 5 or 10 
minutes acquisitions were performed. Events were grouped offline into $3 \mathrm{~s}$ measurements using the time stamps. This process was done in order to verify the detection probability (PD) at a given confidence level (CL). Test were performed at $\mathrm{PD}=90 \%, \mathrm{CL}=95 \%$ according to the prescriptions in ref. 8 .

The small $6 \mathrm{~g} \mathrm{Pu}$ samples produced alarms both for neutrons and for gamma rays satisfying the required PD. Gamma rays spectra from the $\mathrm{Pu}$ samples have been studied making use of the $\mathrm{LaBr}(\mathrm{Ce})$ scintillator, needed to disentangle the complex spectra [9]. Few gamma ray transitions are identified: the lines at $E \gamma=373,414$ and $451 \mathrm{keV}$ which can be attributed to the ${ }^{239} \mathrm{Pu}$ decay and those at $\mathrm{E} \gamma=662$ and $772 \mathrm{keV}$ from the ${ }^{241} \mathrm{Am}$ decay. Moreover, the $\mathrm{E} \gamma=208 \mathrm{keV}$ line is identified, due to the ${ }^{241} \mathrm{Am}$ nucleus as well as to the ${ }^{237} \mathrm{U}$, another daughter nucleus often in secular equilibrium with ${ }^{241} \mathrm{Pu}$. [10]. The intensity ratios are different according with the $\mathrm{Pu}$ sample isotopic composition. Comparable results were also obtained by using a quite large MOX sample containing a large amount of $\mathrm{Pu}(170 \mathrm{~g})$ mixed with uranium oxides $(1011 \mathrm{~g})$. The MOX sample was shielded with $2.5 \mathrm{~cm}$ lead.

Uranium samples with different enrichment were also investigated. The intensity of gamma ray emission from the samples is sufficient to yield an alarm, whereas neutron emission resulted to be only about twice the laboratory background. Consequently long measuring times are required in order to get a neutron alarm with the required confidence level. For the highly enriched sample, the E $\gamma=186 \mathrm{keV}$ transition emitted in the decay of ${ }^{235} \mathrm{U}$ is clearly seen in the gamma ray spectra, whereas for the low enrichment samples two transitions are seen at $E \gamma=767$ and $1001 \mathrm{keV}$ emitted in the decay of the ${ }^{234 \mathrm{~m}} \mathrm{~Pa}$ nucleus which is in secular equilibrium with the ${ }^{238} \mathrm{U}$ nucleus.

As shown in this section, the Pu samples produce neutron as well as gamma ray alarms in the detection system when the dose delivered at the detector surface is 0.5 $\mu \mathrm{Sv} / \mathrm{h}$. In the same condition the $\mathrm{U}$ samples produce also a clear gamma ray alarm, being the neutron emission too weak. In both cases the $\mathrm{LaBr}(\mathrm{Ce})$ gamma ray spectra provide hints on the isotopic composition. However, the photon signature from highly enriched uranium can be easily shielded and it is extremely weak even after $1 \mathrm{~cm}$ lead due to the low energy of the ${ }^{235} \mathrm{U}$ transition and the intrinsic low yield of the ${ }^{238} \mathrm{U}$ one. Consequently the detection of uranium samples in passive mode seems to be difficult in case the sample is shielded with high $\mathrm{Z}$ material. Detection of uranium with active interrogation is the subject of the next section.

\section{ACTIVE INTERROGATIONS}

In active interrogations the associated particle detector signal is also processed in the V1720 card. The alpha particles emitted in the ${ }^{3} \mathrm{H}\left({ }^{2} \mathrm{H},{ }^{4} \mathrm{He}\right) \mathrm{n}$ reaction are indeed detected in a fast $\mathrm{YAP}(\mathrm{Ce})$ scintillator embedded in the EADS SODERN TPA17 neutron generator, coupled to an external HAMAMATSU R1450 PMT. The associated particle detector covers a fraction of solid angle of about $1 \times 10^{-3}$ of $4 \pi$ so that a rate of $10 \mathrm{kHz}$ is expected in the operation of the neutron generator at a total intensity of $10^{7}$ neutron/s, the limit being imposed by the laboratory license. In the above conditions, the spot of the tagged neutron beam produced by the TPA17 generator at the object position, located about $30 \mathrm{~cm}$ from the detector box surface, has been measured to be about $15 \mathrm{~cm}$ in diameter [FWHM]. 
In the active mode operations we stored directly all single events processed by the V1720 card running at a typical total rate of about $50 \mathrm{kHz}$, writing only the most significant part of the digitized signals. Off-line software analyzes the event files reconstructing the coincidence events and the time correlation between detectors. The time interval from the start time of the digitization and a given fraction of the front part of the signal is determined for each detector, correcting for the amplitude effects. This analysis yields a time resolution better than the V1720 sampling bin (4 ns). Laboratory tests using gamma-gamma coincidences with a ${ }^{22} \mathrm{Na}$ source and a fast plastic as trigger detector are $\delta \mathrm{t}=1.15 \mathrm{~ns}[\mathrm{FWHM}]$ for $\mathrm{LaBr}(\mathrm{Ce})$ and $\delta \mathrm{t}=5.4 \mathrm{~ns}$ [FWHM] for $\mathrm{NaI}(\mathrm{Tl})$, respectively, with the lower threshold discrimination set at about $500 \mathrm{keV}$.

In the active interrogation tests the two large (about $2.5 \mathrm{~kg}$, marked as LU25 and LU44) U samples with different enrichment were employed together with a $16.7 \mathrm{~kg}$ iron cylinder, a $6.7 \mathrm{~kg}$ lead brick and a sample of about $10 \mathrm{~kg}$ organic material made of 50\% Plexiglas and 50\% melamine powder. Interrogation runs lasted 10 minutes. Typical results for $U$ samples shows the well known pattern of radioactive emission from ${ }^{238} \mathrm{U}$ at $\mathrm{E} \gamma=767$ and $1001 \mathrm{keV}$ in the $\mathrm{LaBr}(\mathrm{Ce})$ singles. The time distribution of the coincidences exhibits a double peaked structure due to the detection in the $\mathrm{LaBr}(\mathrm{Ce})$ detector of gamma rays and neutrons from the irradiated sample. Finally the energy distribution of the gamma ray in prompt coincidence mode shows no relevant structures that can be used to identify directly the uranium nuclei. The situation is different in irradiating iron, lead or organic material. In this case, obviously, the spectrum of the $\operatorname{LaBr}(\mathrm{Ce})$ detector does not show signatures due to the internal radioactivity of the sample. he coincident spectra of iron and organic exhibit the well known gamma ray transitions of inelastic excitation of iron $(\mathrm{E} \gamma=0.847$ and $\mathrm{E} \gamma=1.238$ $\mathrm{MeV})$ and carbon nuclei $(\mathrm{E} \gamma=4.44 \mathrm{MeV})$ that can be used to identify easily this type of materials [6]. Moreover, the $\operatorname{LaBr}(\mathrm{Ce})$ coincident spectrum of the lead sample shows some structures that can be attributed to transitions in $\mathrm{Pb}$ isotopes as the well known $\mathrm{E} \gamma=2.61 \mathrm{MeV}$ in ${ }^{208} \mathrm{~Pb}$. However the coincident discrete gamma ray spectrum alone seems to be hardly usable to distinguish between $\mathrm{Pb}$ and $\mathrm{U}$.

Consequently we have explored other signatures collected by our detection system.

The time spectrum of the NE213 detector in coincidence with the associated alpha particles shows for each sample a very narrow peak due to prompt coincidences, mainly due to neutron induced gamma rays, well separated by a second larger bump due to secondary neutrons produced by the $14 \mathrm{MeV}$ neutron beam. Such secondary neutrons are associated to fission $(n, f)$ as well as $(n, x n)$ reactions. The pulse-shape discrimination is used, in this case, to discriminate gamma rays and neutrons in addition to the time of flight information. We then derived the number of the detected gamma ray and neutrons from the particle discrimination algorithm after windowing the time difference between the alpha particle and the liquid scintillator signals. This allows a very good discrimination between the two types of events.

The correlation between the number of gamma rays and neutrons detected in the liquid scintillator for each sample normalized to sample weight and the ratio sample/beam area is used to discriminate the different materials, as shown in Fig.1. It is interesting to note that the relative number of detected gamma rays and neutrons for each sample is correlated to the relevant neutron and gamma production cross sections for each elementary sample. The difference between uranium and materials commonly 
used for shielding appears to be very large. As a conclusion, the empirical correlation displayed in Fig. 1 shows that, taking into account sample mass and area, it is possible to discriminate uranium from other materials using only the liquid scintillator data.

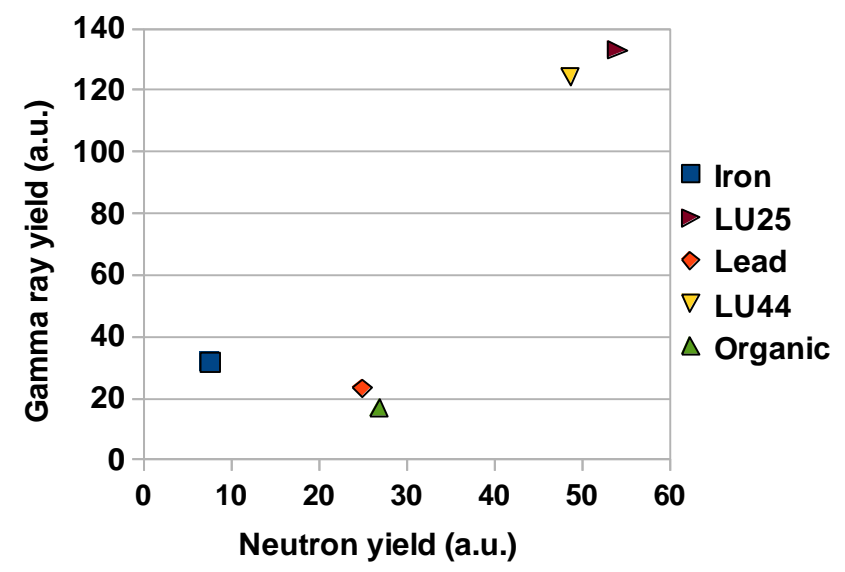

FIGURE 1.

In case of the inspection of a suspect volume, as the case of cargo container, the tagged neutron technique allows to define a voxel inside the total volume. In this case it is questionable that a representation as that in Fig. 1 could be applied to search for uranium. Consequently, new observables are required to evidence the presence of fissile material. In such case the gamma ray spectrum of $\mathrm{LaBr}(\mathrm{Ce})$ easily identifies the presence of organic or iron based material through the well known gamma lines. In order to obtain a discrimination plot, triple coincidences between the associated alpha particle, the liquid scintillator and the large volume $\mathrm{NaI}(\mathrm{Tl})$ detector were analyzed. The idea is to verify whether the multiplicity of gamma rays and neutrons emitted in the fission of ${ }^{238} \mathrm{U}$ provides a signature different from that of $\mathrm{Pb}$ nuclei for which neutron multiplication is due only to $(\mathrm{n}, \mathrm{xn})$ reactions.

In order to obtain a parameter without need of a normalization, the number of triple coincidences (YAP:Ce-NE213-NaI(Tl)) is divided by the number of double coincidences between (YAP:Ce-NE213). This analysis is repeated twice for gamma rays or neutrons identified in the liquid scintillator. In Fig. 2 the data obtained in this way are plotted in a 2-D representation of neutron events against gamma events for the different samples explored in this work. This empirical representation results in the grouping of the sample in three regions. Lead and organic material exhibit a high probability of triple coincidence for gamma ray events (in the NE213 detector) but low for events involving neutrons. Iron is characterized by a larger probability for neutron coincidence events compared to previous samples but lower for gamma rays. Finally the two uranium samples have the largest probability for neutron triple events but an intermediate value for the gamma rays compared to all previous samples. As a result, clear distinction between uranium and other samples is obtained. 


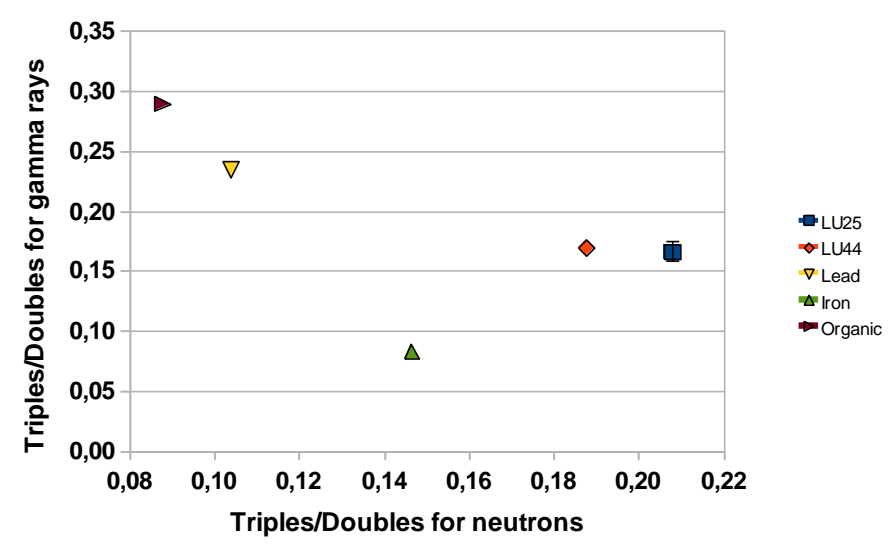

FIGURE.2

\section{SUMMARY AND CONCLUSIONS}

The detection of special nuclear material has been tested with the mobile SMANDRA inspection system both as a high sensitivity passive spectroscopic tool and as a complete active inspection device using tagged neutrons.

The detection of plutonium samples seems to be possible with passive interrogation even in case of small samples, due to the yield of gamma ray and neutrons. On the other hand it is well known that detection of uranium is much more difficult on one side because of the low neutron yield of this material, on the other side since the gamma ray yield of highly enriched $U$ samples could be easily shielded. In this case active interrogation is needed.

Results reported in this paper show that the SMANDRA inspection system is able to provide signatures for the discrimination of uranium against heavy metals (as lead) by looking at the absolute gamma and neutron yield in coincidence with tagged neutrons or at correlations between detectors. It is worth mentioning that the SMANDRA system is a mobile multi-purpose spectrometric system not specifically designed to detect SNM. However the results reported might be implemented in future portable systems specifically designed to detect SNM in active mode.

\section{REFERENCES}

1. S. Pesente et al., Nucl. Instrum. and Meth. B241 (2005) 743-747.

2. D. Cester et al., to be published in the IEEE Proccedings of the 2011 2nd International Conference on Advancements in Nuclear Instrumentation, Measurement Methods and their Applications, Gand (Belgium) June 2011

3. R. Nicolini et al., Nucl. Instrum. and Meth. A582 (2007), 554-561 and references therein.

4. B.D. Milbrath et al., Nucl. Instrum. and Meth. A572 (2007) 774-784.

5. W. ElKanawati et al., Applied Radiation and Isotopes 69 (2011) 732-743.

6. R. T. Kouzes et al., Nucl. Instrum. and Meth. A 584 (2008) 383-400.

7. R. T. Kouzes et al., Nucl. Instrum. and Meth. A 587 (2008) 89-100

8. D. Gilliam et al., J. Res. Natl. Inst. Stand. Technol. 114 (2009) 195-199.

9. J. P. Sullivan et al., Journal of Radioanalytical and Nuclear Chemistry, Vol. 276, (2008) 699-705

10. high resolution gamma ray spectra are available at the site http://www.radiochemistry.org/periodictable/gamma_spectra/ 